Research Paper

\title{
Individual risk of post-ivermectin serious adverse events in subjects infected with Loa loa
}

\author{
Cédric B. Chesnais ${ }^{\mathrm{a}, \mathrm{b}, \mathrm{c}}$, Sébastien D. Pion ${ }^{\mathrm{a}, \mathrm{b}, \mathrm{c}}$, Charlotte Boulléb,c,d,e , Jacques Gardon $^{\mathrm{f}}$, \\ Nathalie Gardon-Wendel ${ }^{g}$, Joël Fokom-Domgue ${ }^{\mathrm{h}}$, Joseph Kamgno ${ }^{\mathrm{i}, \mathrm{j}}$, Michel Boussinesq ${ }^{\mathrm{a}, \mathrm{b}, \mathrm{c}, *}$ \\ a UMI 233, Institut de Recherche pour le Développement (IRD), Montpellier, France \\ ${ }^{\mathrm{b}}$ Université Montpellier, Montpellier, France \\ ${ }^{\mathrm{c}}$ INSERM Unité 1175, Montpellier, France \\ d Infectious Disease Department, Montpellier University Hospital, Montpellier, France \\ e Institut de Recherche pour le Développement (IRD), Montpellier, France \\ ${ }^{\mathrm{f}} H S M, I R D, C N R S$, University of Montpellier, Montpellier, France \\ ${ }^{\mathrm{g}}$ Antenne ORSTOM auprès du Centre Pasteur, Yaoundé, Cameroon \\ ${ }^{\mathrm{h}}$ Department of Epidemiology, The University of Texas MD Anderson Cancer Center, Houston, TX, USA \\ ${ }^{\mathrm{i}}$ Professor, Centre for Research on Filariasis and other Tropical Diseases, Yaounde, Cameroon \\ ${ }^{\mathrm{j}}$ Department of Public Health, Faculty of Medicine and Biomedical Sciences, University of Yaounde I, Yaounde, Cameroon
}

\section{A R T I C L E I N F O}

\section{Article History:}

Received 12 August 2020

Revised 2 September 2020

Accepted 22 September 2020

Available online 10 October 2020

\section{Keywords:}

Loiasis

Serious adverse events

Africa

\begin{abstract}
A B S T R A C T
Background: Implementation of onchocerciasis elimination programmes has been delayed in Central Africa because of the risk of ivermectin-related serious adverse events (SAEs) in individuals with high Loa loa microfilarial densities (MFD). We developed the first statistical models enabling prediction of SAE risk in individuals with a given MFD.

Methods: We used individual participant data from two trials conducted in loiasis-onchocerciasis co-endemic areas in Cameroon. among the 10506 ivermectin-treated subjects included in the analysis, 38 (0.36\%) developed an ivermectin-related SAE. To predict individual-level risk of SAE, we developed mixed multivariate logistic models including subjects' sex, age, pre-treatment $L$ loa and Mansonella perstans MFDs, and study region.

Findings: The models predicted that regardless of sex, about $1 \%$ of people with $20000 \mathrm{~L}$ loa microfilariae per millilitre of blood $(\mathrm{mf} / \mathrm{mL}), 10 \%$ of people with $50000 \mathrm{mf} / \mathrm{mL}$ and about one third of those with $100000 \mathrm{mf} / \mathrm{mL}$ will develop an SAE. For a given MFD, males have a three-fold higher risk of developing an SAE than females.

Interpretation: By enabling the prediction of post-ivermectin SAE risk in communities with known distribution of $L$ loa MFDs, our results can guide decisions on the choice of ivermectin-based treatment strategies. They also predict that 37 SAEs were prevented in 2015 by using a Test-and-Treat strategy in the Okola District of Cameroon.

Funding: UNDP/World Bank/WHO Special Programme for Research and Training in Tropical Diseases; Institut de Recherche pour le Développement; Mectizan Donation Program; Bill \& Melinda Gates Foundation.

(c) 2020 The Authors. Published by Elsevier Ltd. This is an open access article under the CC BY-NC-ND license
\end{abstract} (http://creativecommons.org/licenses/by-nc-nd/4.0/)

\section{Introduction}

For more than 20 years, the implementation of onchocerciasis control and elimination programmes in Central Africa has been hampered by loiasis (Loa loa filariasis). This is because ivermectin (IVM), distributed via mass treatment to eliminate onchocerciasis, can induce a potentially fatal encephalopathy in subjects with a $L$ loa

\footnotetext{
* Corresponding author at: Institut de Recherche pour le Développement (UMI 233), 911 avenue Agropolis, BP 64501, 34394 Montpellier cedex 5, France.

E-mail address: michel.boussinesq@ird.fr (M. Boussinesq).
}

microfilarial density (MFD) exceeding 30000 microfilariae per millilitre of blood $(\mathrm{mf} / \mathrm{mL})$ [1]. As a result, in settings where loiasis is present, IVM is distributed only in onchocerciasis meso- and hyperendemic areas, where the benefit of treatment outweighs the risk of serious adverse events (SAE). In these areas, the standard community-directed treatment with IVM (CDTI) is complemented with a specific surveillance system to identify and manage any SAE case occurring. However, the continued exclusion of hypoendemic areas may delay or compromise onchocerciasis elimination. Alternative treatment strategies (ATS) to safely treat populations in 


\section{Research in context}

\section{Evidence before this study}

In Central Africa, loiasis hinders mass distribution of ivermectin against onchocerciasis because subjects with high Loa loa microfilarial densities can develop serious adverse events after treatment with ivermectin. In areas where onchocerciasis is hypoendemic and where loiasis is coendemic, the risk of serious adverse events during mass distribution of ivermectin might outweigh the benefit from treatment, and programme managers have to decide whether they should use alternative approaches such as "Test-and-treat" strategies. Currently, only estimates of the relative risks to develop a serious adverse event above a given microfilarial density threshold exist, which is insufficient to make evidence-based decisions between strategies with very different cost implications.

\section{Added value of this study}

The new models allow to estimate the risk of developing a postivermectin serious adverse event of individuals with a given $L$ loa microfilarial density. In settings where the distribution of $L$ loa microfilarial density across individuals is known, the number of serious adverse event cases that are expected during mass distribution of ivermectin and by extension, the number of serious adverse events that would be prevented using a "Test-and-treat" strategy, can be estimated.

\section{Implications of all the available evidence}

The results of this study allow programme managers and decision-makers to better predict the risk of post-ivermectin serious adverse events at the community level when the drug is distributed in areas where loiasis is endemic and the distribution of $L$ loa microfilarial densities has been determined. Accurate quantification of this risk will contribute to the development of the most suitable and cost-effective treatment strategies for these areas and therefore, help prevent the occurrence of serious adverse events during treatment campaigns.

onchocerciasis hypoendemic areas that are coendemic for loiasis have been proposed, including a "Test-and-treat" (TNT) approach involving identification of subjects at risk of post-IVM SAEs, and providing IVM only to subjects whose MFD is below the risk threshold $[2,3]$. However, this strategy is more costly than CDTI [4], and concerns about its scalability and sustainability have been raised. Key stakeholders have suggested that the standard CDTI with enhanced surveillance could be implemented in onchocerciasis hypoendemic areas where the community risk of SAE occurrence is below a given threshold [5]. To accurately assess this community risk, two types of information are required: the $L$ loa microfilarial density distribution in the targeted population, and the individual probability to develop an SAE for a given MFD.

In this study, we developed and assessed multivariate models to estimate the probability of an individual with a given MFD to develop a post-IVM SAE. This critical information will aid decision-makers in choosing the most appropriate treatment strategy for onchocerciasis hypoendemic areas.

\section{Method}

\subsection{Study population}

Data used in the analyses were collected as part of two clinical trials. The first was carried out in 1995-1996 in the Lekie division
(Centre Region) of Cameroon, to determine the incidence of post-IVM SAEs in a loiasis-endemic area, and the $L$ loa MFD threshold above which SAEs can occur [1] This study included 17877 subjects aged $\geq 5$ years living in 106 villages, including 5550 IVM-naïve subjects aged $\geq 15$ years whose pre-treatment $L$ loa MFD was measured. The second trial was carried out in 2005 in the Lom-et-Djerem and HautNyong divisions (East Region) of Cameroon to evaluate whether coinfection with Plasmodium sp. is a risk cofactor for Loa-related postIVM SAE [6]. This study included 4956 IVM-naïve subjects aged $\geq 13$ years living in 74 villages. Both studies were approved by the Cameroon National Ethics Committee for Research in Human Health $[1,6]$. For the study conducted in the East Region, all voluntary participants provided a signed informed consent. For the Lekie study supported by the WHO-TDR in 1995-1996, the Ministry of Public Health of Cameroon considered that the study area had to be treated with ivermectin anyway, and that no consent form had to be signed by the voluntary participants. This study follows the STROBE guidelines.

\subsection{Parasitological examination}

Details about the parasitological examination are provided in the original publications [1,6]. Briefly, in both trials a calibrated $(50 \mu \mathrm{L})$ thick blood smear (TBS) from a finger prick obtained between 10:00 AM and 4:00 PM was performed to measure the L loa and Mansonella perstans (another filaria which is endemic in these areas) MFDs. Within $48 \mathrm{~h}$, the slides were dehaemoglobinized and stained with Giemsa. The TBS were read by experienced microscopists and individual MFDs expressed in $\mathrm{mf} / \mathrm{mL}$ of blood.

\subsection{Treatment and monitoring of serious adverse events}

In both trials, IVM was given orally to eligible individuals at a standard dose of $150 \mu \mathrm{g} / \mathrm{kg}$ of body weight and an active surveillance procedure to track adverse events was established in all communities for the 7 days following treatment. SAEs were considered related to IVM treatment when the pre-treatment $L$ loa MFD exceeded $10,000 \mathrm{mf} / \mathrm{mL}$ with consistent symptoms (e.g. headache, asthenia, fever) [7], or when the patient presented haemorrhages of the palpebral conjunctiva after treatment [8]. Patients were classified as having a non-neurological SAE when they had functional impairment that required full-time assistance for at least one week; these patients had no objective neurological signs, but were exhausted and unable to stand up and walk without support, remaining in bed or in armchairs; some were hospitalized in classical inpatient structures when available or required significant outpatient care when impossible due to local constraints. Subjects classified as having neurological SAEs developed disorders of consciousness and objective neurological signs and were admitted to the hospital for appropriate management.

\subsection{Statistical analyses}

\subsubsection{General model}

In this analysis we included both non-neurological and neurological ivermectin-related SAEs, subsequently referred to as SAEs. The outcome variable was the occurrence of an SAE and the independent variables were the subjects' age, sex, pre-treatment $L$ loa and $M$ perstans MFDs, and the study site (Centre or East region). First, bivariate logistic models were constructed to determine the most appropriate transformation to apply to the three continuous covariates (age, $L$ loa MFD, and $M$ perstans MFD) to model their association with the occurrence of an SAE. These variables were considered alternately as categorical and continuous variables. When described as categorical variables, these covariates were classified as follows: age (13-20, 21-30, 31-50, > 50), L loa MFD (0-10,000, 10,001-30,000, $30,001-50,000,>50,000)$, and $M$ perstans MFD $(0,1-50,51-100$, 
$101-300,>300)$. When described as continuous variables, these three covariates were used either without transformation (assuming a linear relationship) or after quadratic, logarithmic or fractional polynomials (FP) of order 1 or 2 (FP1 or FP2) transformations - each of these transformations assumes a (different) non-linear relationship between the continuous covariate and the outcome. To select the most adapted transformation, we used the Akaike information criteria (AIC). After identification of the best transformation for each independent variable, we designed four different models (after testing for a random-effect at the village level) that included an increasing number of variables (models called A, B, C, and D): (A) L loa MFD only; (B) $L$ loa MFD and sex; (C) $L$ loa MFD, sex and age; and (D) $L$ loa MFD, sex, age and $M$ perstans MFD ("full model"). A fifth model adding the "Region" variable (Centre vs. East) to the "full model" was also developed to determine whether the estimated risk differed between regions. The significance of all possible interaction terms as well as the village-level random effect were assessed using the likelihood ratio's test. Then, models A and B were used to estimate the individual risk of developing a post-IVM SAE for subjects with 5000, 10,000, 20,000, 30,000,40,000,50,000, 100,000, 150,000 and 200,000 L loa $\mathrm{mf} / \mathrm{mL}$.

\subsubsection{Semi-external validation}

A semi-external validation was performed using bootstrap and 0.632 bootstrap procedures. Briefly, 1000 resampling iterations generated both a training and a testing set. Regression models were fitted on the training set and predictions were estimated on the outs of bag (testing set), allowing to calculate the estimated number of ESGs according to the procedure described above as compared to the actual number of ESGs observed in the testing sample. The median difference is reported in absolute counts and percentage variation, along with their interquartile range (IQR) and the 0.025 and 0.975 quantiles. For each model, medians and interquartile ranges of each $\beta$ coefficient obtained through the 0.632 bootstrap procedure are reported.

\subsubsection{Extrapolation to an external population}

In addition, we used these models to estimate the number of SAEs prevented in 2015 in a health district of Cameroon where the efficacy and feasibility of the TNT strategy were assessed [2]. In this study, the
L loa MFD of 16259 subjects was measured by both calibrated $(50 \mu \mathrm{L})$ TBS and a rapid diagnostic tool called LoaScope (see details below), and all those with more than $20,000 \mathrm{mf} / \mathrm{mL}$ (as assessed with the LoaScope) were excluded from IVM treatment (340 subjects). To estimate the number of SAEs prevented in this study, we applied models A and B to the distribution of $L$ loa MFDs measured in this population by TBS, which remains the standard method to measure MFDs. All analyses were performed using Stata ${ }^{\square}$ (StataCorp, College Station, Texas, TX, USA).

\subsection{Role of the funding source}

The funders of the clinical trials whose data were used to develop the models and to estimate the number of SAEs prevented by a TNT approach had no role in the study design, the interpretation of the results, or the writing of this manuscript. The present work was conducted without additional financial support. The corresponding author had full access to all the data collected in the trials. The first and corresponding authors had final responsibility for the decision to submit the publication.

\section{Results}

\subsection{Population characteristics and number of serious adverse events}

All 10506 subjects from the 1995-1996 and 2005 studies, with known $L$ loa MFD, were included in the analysis. among these, 38 subjects had developed an SAE (0.36\%), including two with non-fatal neurological SAE. $L$ loa MFDs in subjects who experienced an SAE ranged between 5620 and $182,400 \mathrm{mf} / \mathrm{mL}$. Only three subjects with SAEs had a $L$ loa MFD $<30,000 \mathrm{mf} / \mathrm{mL}$ (5620, 23,720 and 26,820 mf/ $\mathrm{mL}$, respectively) and the MFDs of the neurological cases were 50,520 and $152,940 \mathrm{mf} / \mathrm{mL}$. The mean age of those who developed an SAE was slightly higher than among other participants (Table 1). SAEs were more frequent in males than in females.

\subsection{Categorisation and transformation of the continuous variables}

Age was transformed using FP2 (AIC 492-46) (appendix p 1). L loa and $M$ perstans MFDs were first included as FP2 (AIC 214.27 and

Table 1

Description of the study population.

\begin{tabular}{|c|c|c|c|c|c|}
\hline & & All subjects N (100\%) & Subjects without SAE N (\%) & Subjects with SAE N (\%) & p value* \\
\hline Mean Age (SD) & & $35 \cdot 4(18 \cdot 2)$ & $35 \cdot 4(18 \cdot 2)$ & $40 \cdot 8(16 \cdot 2)$ & $0 \cdot 024$ \\
\hline \multirow[t]{4}{*}{ Age (years) (no. and\%) } & $13-20$ & $3147(30 \cdot 0)$ & $3145(30 \cdot 0)$ & $2(5 \cdot 3)$ & $0 \cdot 001$ \\
\hline & $21-30$ & $1984(18 \cdot 9)$ & $1975(18 \cdot 9)$ & $9(23 \cdot 7)$ & \\
\hline & $31-50$ & $2951(28 \cdot 1)$ & $2932(28 \cdot 0)$ & $19(50 \cdot 0)$ & \\
\hline & $>50$ & $2424(23 \cdot 1)$ & $2416(23 \cdot 1)$ & $8(21 \cdot 0)$ & \\
\hline \multirow[t]{2}{*}{ Sex (no. and\%) } & Male & $5139(48 \cdot 9)$ & $5109(48 \cdot 8)$ & $30(79 \cdot 0)$ & $<0.0001$ \\
\hline & Female & $5367(51 \cdot 1)$ & $5359(51 \cdot 2)$ & $8(21 \cdot 0)$ & \\
\hline \multirow[t]{2}{*}{ Region } & Centre & $5550(52 \cdot 8)$ & $5530(52 \cdot 8)$ & $20(52 \cdot 6)$ & 0.554 \\
\hline & East & $4956(47 \cdot 2)$ & $4938(47 \cdot 2)$ & $18(47 \cdot 4)$ & \\
\hline \multirow[t]{2}{*}{ Presence of $L$ loa $\mathrm{mf}$} & No & $7534(71 \cdot 7)$ & $7534(72 \cdot 0)$ & 0 & $<0.0001$ \\
\hline & Yes & $2972(28 \cdot 3)$ & $2934(28 \cdot 0)$ & $38(100)$ & \\
\hline \multirow[t]{4}{*}{ L loa MFD (mf/mL) } & $0-10000$ & $9781(93 \cdot 1)$ & $9780(93 \cdot 4)$ & $1(2 \cdot 63)$ & $<0.0001$ \\
\hline & $10001-30000$ & $481(4 \cdot 6)$ & $479(4 \cdot 6)$ & $2(5 \cdot 3)$ & \\
\hline & $30001-50000$ & $133(1 \cdot 3)$ & $126(1 \cdot 2)$ & $7(18 \cdot 4)$ & \\
\hline & $>50000$ & $111(1 \cdot 1)$ & $83(0 \cdot 8)$ & $28(73 \cdot 7)$ & \\
\hline \multirow[t]{2}{*}{ Presence of $M$ perstans $\mathrm{mf}$} & No & $8042(76 \cdot 6)$ & $8026(76 \cdot 7)$ & $16(42 \cdot 1)$ & $<0.0001$ \\
\hline & Yes & $2464(23 \cdot 4)$ & $2442(23 \cdot 3)$ & $22(57 \cdot 9)$ & \\
\hline \multirow[t]{5}{*}{ M perstans MFD (mf/mL) } & 0 & $8042(76 \cdot 5)$ & $8026(76 \cdot 7)$ & $16(42 \cdot 1)$ & $<0.0001$ \\
\hline & $1-50$ & $829(8.9)$ & $826(7 \cdot 9)$ & $3(7 \cdot 9)$ & \\
\hline & $51-100$ & $527(5 \cdot 0)$ & $524(5 \cdot 0)$ & $3(7 \cdot 9)$ & \\
\hline & $101-300$ & $553(5 \cdot 3)$ & $546(5 \cdot 2)$ & $7(18 \cdot 4)$ & \\
\hline & $>300$ & $555(5 \cdot 3)$ & $546(5 \cdot 2)$ & $9(23 \cdot 7)$ & \\
\hline
\end{tabular}

SAE: severe adverse events; SD: standard deviation; MFD: microfilarial density.

* Mean age was compared with the Mann-Whitney test; sex, region, presence of $L$ loa mf, presence of $M$ perstans $\mathrm{mf}, L$ loa MFD and $M$ perstans MFD variables were compared with $\chi 2$ test. 
473.98, respectively). However, we considered that FP2 transformation of $L$ loa MFD did not provide realistic estimates because it led to a decrease in the risk of SAE for the highest MFDs (figure in appendix $\mathrm{p} 2$ ). We attribute this to the fact that when the MFDs are extremely high, accurate quantification of $\mathrm{mf}$ present on the slide (some of which may be overlapping) may be challenging for the microscopist. Consequently, MFDs above $150000 \mathrm{mf} / \mathrm{mL}$, found in only 10 subjects, were grouped together, with a value of $150,000 \mathrm{mf} / \mathrm{mL}$, and the $L$ loa MFD was transformed using FP1.

\subsection{Multivariate modelling}

Table 2 presents the results of the four multivariate models and Fig. 1 shows the predicted probabilities to develop an SAE for a given $L$ loa MFD (for model A, and see in appendix p 3 the figures with the four models). None of the models indicated significant random effects at the village level (Table 2, last line). With all other variables there was no evidence of a significant difference in SAE risk between the East and Centre regions (adjusted Odds-Ratio 1·71, $p=0 \cdot 193$ ). A significant interaction was detected between $M$ perstans MFD and age $(p=0.0275)$. However, this $\mathrm{p}$ value increased to 0.083 after adjustment on $L$ loa MFD and thus, no interaction term was included in the models.

\subsection{Predicted probability of developing a serious adverse event}

Equations describing the transformations applied to the independent variables are presented in the appendix $\mathrm{p} 4$. Table 3 presents the probabilities of developing an SAE for various given $L$ loa MFDs, as predicted by model A (i.e. taking into account only the $L$ loa MFD), and model B (including both $L$ loa MFD and sex). It shows that the risk remains below $1 \%$ for MFDs $<20,000 \mathrm{mf} / \mathrm{mL}$ and increases substantially to 77\% when the MFD reaches $200000 \mathrm{mf} / \mathrm{mL}$. Males have a significantly higher adjusted odds-ratio when compared to females: $3 \cdot 07(p=0 \cdot 011), 2 \cdot 81(p=0 \cdot 020)$ and $3 \cdot 18(p=0 \cdot 015)$ for models $\mathrm{B}, \mathrm{C}$ and $D$, respectively. Fig. 2 illustrates this difference when applying model D. Age (see appendix p 5) and $M$ perstans MFD have a non- or marginally significant influence on the probability of developing an SAE. When age was treated as a categorical rather than a continuous variable, we observed a marginally increased risk only in those aged $30-40$ years $(p=0 \cdot 029)$ (see appendix p 5). Finally, the equation of the probability of developing an SAE according to the individual $L$ loa MFD (from the model $A$ ) is the following:

Probability $_{S A E}=\frac{\exp ^{\left[-9.74+2.54 \times\left(\ln \frac{\operatorname{Loa}+2.22216796875}{100000}\right)+3.658185875\right]}}{1+\exp ^{\left[-9.74+2.52 \times\left(\ln \frac{\operatorname{Loa}+2.22216796875}{100000}\right)+3.658185875\right]}}$

\subsection{Semi-external validation}

Table 4 shows the details of the results of the misclassification estimations after bootstrap procedures. The median difference between estimated and observed SAEs were $-0 \cdot 13,-0 \cdot 18,0 \cdot 05$, and $0 \cdot 22$ cases, respectively for the model A, B, C, and D, respectively, corresponding to a median variation of $-0 \cdot 91 \%,-1 \cdot 33 \%, 0 \cdot 45 \%$, and $1 \cdot 75 \%$, respectively, when reported to the number of SAEs. Medians and IQR of each $\beta$-coefficient obtained through the 0.632 bootstrap procedure are reported in the Table 5.

\subsection{Application to a different population in which MFDs were measured as part of the evaluation of a "Test-and-treat" strategy}

As part of a TNT campaign conducted in 2015 in the Okola District in Cameroon, MFD was measured in 16259 subjects [2]. Using the L loa MFD as the only predictor (model A), we estimated that the exclusion of 340 subjects with $>20000 \mathrm{mf} / \mathrm{mL}$ from IVM treatment prevented 37 (95\% CI 24-50) cases of SAE. Using both $L$ loa MFD and sex as predictor variables (model B), we estimated the number of SAE cases prevented at 36 (95\% CI 19-52). These estimates suggest that more than $10 \%$ of those who had been excluded from IVM treatment during the TNT campaign would have developed an SAE if they had received the drug. Based on the data obtained in the previous trial conducted in the same region [2], some of these SAEs would have been potentially fatal neurological SAEs.

\section{Discussion}

This paper presents the first approach ever developed to estimate the probability of a post-IVM SAE in a subject with a given $L$ loa MFD. Our results corroborate previously published estimates of the relative risk of SAEs across groups with different MFD levels [1,6], but represent a significant extension allowing the quantification of individual risk. In doing so, they provide a framework enabling a direct assessment of the risks associated with IVM mass administration and of the benefits of alternative onchocerciasis control strategies such as TNT. The models developed in this study were based on data from the only two population-level studies where post-IVM adverse events were monitored actively in subjects with known $L$ loa MFD. For obvious ethical reasons, further studies of this type could not be conducted.

Our results confirm that the primary determinant of the probability of developing a post-IVM SAE is the L loa MFD. Importantly, the fact that the risk estimates did not differ between the Lekie and Centre regions, as well as the absence of a significant random effect at

Table 2

Multivariable fractional polynomial models to estimate the probability of developing a severe adverse event.

\begin{tabular}{|c|c|c|c|c|c|c|c|c|}
\hline & \multicolumn{2}{|c|}{ Model with L loa MFD alone } & \multicolumn{2}{|c|}{ Adjusted model with sex } & \multicolumn{2}{|c|}{ Adjusted model with age and sex } & \multicolumn{2}{|c|}{ Full adjusted model } \\
\hline & Coefficients (95\% CI) & $\mathrm{p}$ value & Coefficients (95\% CI) & $\mathrm{p}$ value & Coefficients (95\% CI) & $\mathrm{p}$ value & Coefficients (95\% CI) & p value \\
\hline $\operatorname{Loa}^{F P}$ & $2 \cdot 52(1 \cdot 94-3 \cdot 10)$ & $<0.0001$ & $2 \cdot 47(1 \cdot 90-3 \cdot 05)$ & $<0.0001$ & $2 \cdot 46(1 \cdot 88-3 \cdot 04)$ & $<0.0001$ & $2 \cdot 52(1 \cdot 91-3 \cdot 12)$ & $<0.0001$ \\
\hline $\operatorname{Age}_{1}^{F P}$ & & & & & $-1 \cdot 92(-5 \cdot 66-1 \cdot 82)$ & $0 \cdot 315$ & $-1 \cdot 74(-5 \cdot 63-2 \cdot 16)$ & $0 \cdot 382$ \\
\hline $\operatorname{Age}_{2}{ }^{F P}$ & & & & & $13 \cdot 36(-1 \cdot 23-27 \cdot 95)$ & 0.073 & $15 \cdot 33(-0.03-30 \cdot 68)$ & 0.050 \\
\hline Sex & & & $1 \cdot 12(0 \cdot 26-1 \cdot 98)$ & $0 \cdot 011$ & $1.03(0 \cdot 16-1 \cdot 91)$ & $0 \cdot 020$ & $1 \cdot 16(0 \cdot 23-2 \cdot 08)$ & $0 \cdot 015$ \\
\hline Mansonella $_{1}^{F P}$ & & & & & & & $0.02(-0.09-0.13)$ & $0 \cdot 772$ \\
\hline Mansonella $_{2}^{F P}$ & & & & & & & $0 \cdot 30(-0 \cdot 51-1 \cdot 10)$ & $0 \cdot 469$ \\
\hline Intercept & $-9 \cdot 74(-11 \cdot 55--7 \cdot 93)$ & & $\begin{array}{c}-10 \cdot 35(-12 \cdot 26- \\
-8 \cdot 44)\end{array}$ & $<0.0001$ & $-9 \cdot 91(-22 \cdot 86--7 \cdot 96)$ & $<0.0001$ & $\begin{array}{c}-10 \cdot 13(-12 \cdot 22- \\
-8 \cdot 04)\end{array}$ & $<0.0001$ \\
\hline AIC & $216 \cdot 49$ & & $211 \cdot 67$ & & $211 \cdot 26$ & & $205 \cdot 45$ & \\
\hline Deviance & $210 \cdot 49$ & & $205 \cdot 67$ & & $199 \cdot 23$ & & $189 \cdot 45$ & \\
\hline $\begin{array}{l}\text { Predicted number and } \\
\text { 95\% CI }\end{array}$ & $38 \cdot 0(27 \cdot 6-48 \cdot 4)$ & & $38 \cdot 0(27 \cdot 8-48 \cdot 3)$ & & $38 \cdot 0(27 \cdot 8-48 \cdot 2)$ & & $38 \cdot 0(28 \cdot 0-48 \cdot 0)$ & \\
\hline $\begin{array}{l}\text { Random effect on vil- } \\
\text { lages ( } p \text { values) }\end{array}$ & $0 \cdot 250$ & & $0 \cdot 296$ & & $0 \cdot 317$ & & $0 \cdot 298$ & \\
\hline
\end{tabular}

MFD: microfilarial density; CI: confidence interval; FP: fractional polynomials; AIC: Akaike information criterion. 
(a) Loa loa

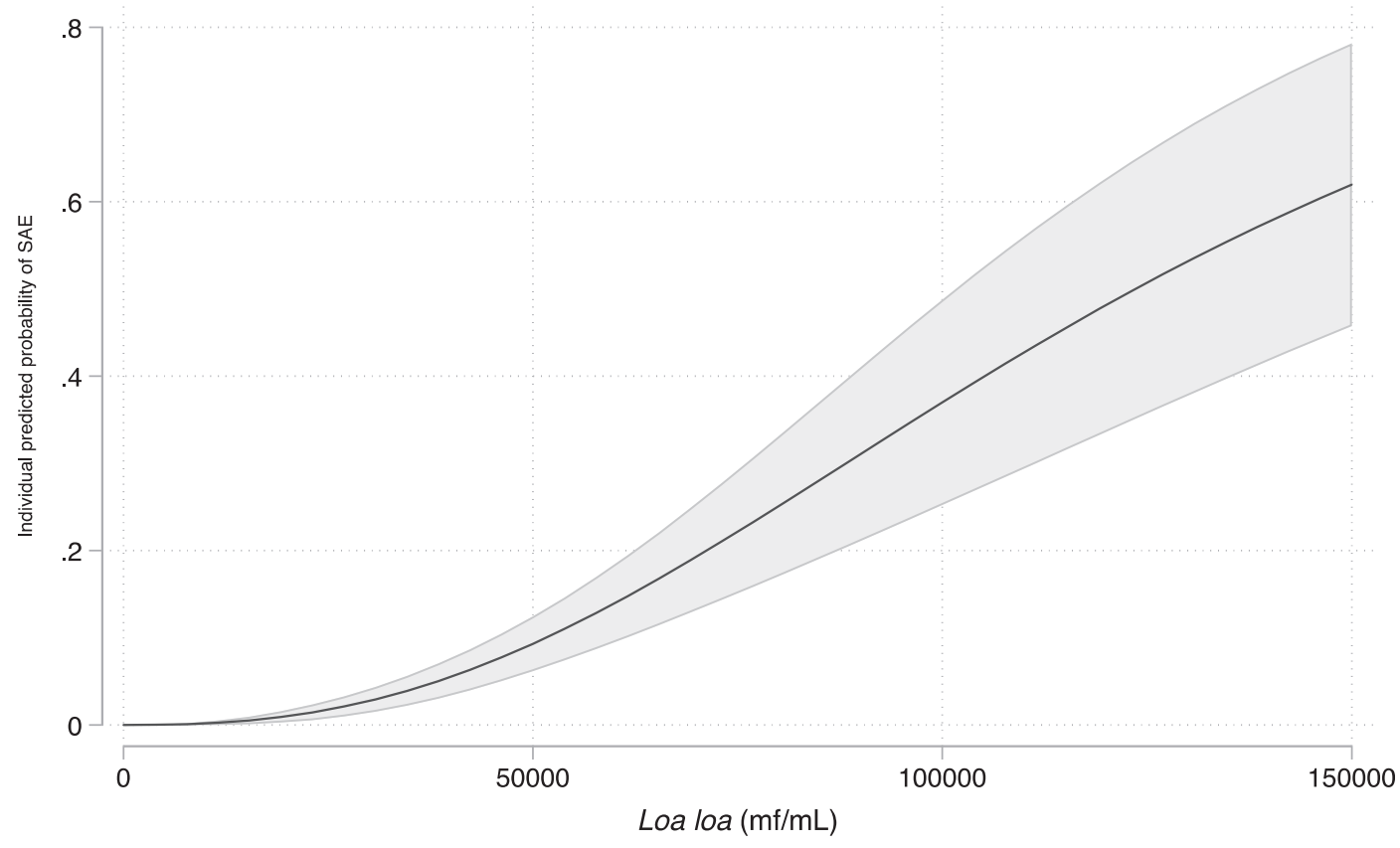

Fig. 1. Predicted probabilities of developing a post-ivermectin severe adverse event according to the individual $L$ loa microfilarial density, overall.

the village level, indicate that the results are consistent across different geographical areas. In addition, male sex was found to be associated with a three-fold increased risk of developing a SAE. This might be due to differential lifestyle habits or coinfections, hormonal influences, physiological features or genetic factors.

In addition to $L$ loa MFD and sex, we also explored the influence of a number of potential confounders on SAE risk, such as age and $M$ perstans MFD (included in models $C$ and $D$ ). These factors had a marginally significant effect, if any, on SAE risk. Thus, consideration of $L$ loa MFD either alone or in conjunction with sex is sufficient to produce accurate estimates of the expected number of SAEs in a population with a known MFD distribution. Interestingly, the estimated numbers of SAEs prevented during the TNT study were similar when applying models A or B (37 and 36 cases, respectively). This suggests that inclusion of sex in the model does not significantly impact the risk estimates at a community level when the male-to-female ratio is close to 1 in the treated population (the proportion of males was $48 \%$ in the TNT study). Therefore, the model including $L$ loa MFD alone should be preferred to the model adjusting on sex when the sex-ratio in the target population is known and close to 1 .

When the distribution of the $L$ loa MFD in the population to be treated with IVM has been assessed, the formulas obtained from the models can be used to accurately estimate the risk of SAE in the community as an expected percentage (with confidence intervals) or absolute numbers of expected SAE cases (with standard deviation). Such risk estimates at the community level can help onchocerciasis programme managers decide which strategy between MDA (which is more affordable but associated with a quantified risk of SAEs), and TNT (which is a safer but more expensive strategy) is most suitable for the target population.

Our models predict that the probability of developing an SAE for a subject with a $L$ loa MFD below $20000 \mathrm{mf} / \mathrm{mL}$ is low (1\%) but not nil. This is due to the fact that one case of SAE was reported in a participant with $5620 \mathrm{~L}$ loa $\mathrm{mf} / \mathrm{mL}$ (in the data base we used, 105 subjects out of 10506 had an MFD between 5000 and $6000 \mathrm{mf} / \mathrm{mL}$ ). This 'outlier' was a 50-year-old male from the East Region, who had fever, headaches, very severe bilateral leg pain, and haemorrhages of the palpebral conjunctiva (a sign frequently associated with post-IVM SAEs)[8] leading the team to hospitalize him before the result of his TBS became available. Although the potential role played by $L$ loa and other cofactors was unclear in this case, the fact that $L$ loa MFD $<20$ $000 \mathrm{mf} / \mathrm{mL}$ can be associated with the development of a clinical condition leading to hospitalization cannot be ignored. However, as a possible explanation for this outlier, it should be noted that in the

Table 3

Probability of developing a post-ivermectin SAE for a given individual $L$ loa microfilarial density (MFD).

\begin{tabular}{|c|c|c|c|c|c|c|c|c|}
\hline \multirow[t]{3}{*}{ L. loa MFD (mf/mL) } & \multicolumn{2}{|c|}{ Model A with $L$ loa MFD as single predictor } & \multicolumn{6}{|c|}{ Model B with both $L$ loa MFD and sex as predictors } \\
\hline & \multirow[b]{2}{*}{$\operatorname{Prob}(\mathrm{SAE})$} & \multirow[b]{2}{*}{$(95 \% \mathrm{CI})$} & \multicolumn{2}{|c|}{ Probability for both sexes combined } & \multicolumn{2}{|c|}{ Probability for males } & \multicolumn{2}{|c|}{ Probability for females } \\
\hline & & & Prob(SAE) & $(95 \% \mathrm{CI})$ & $\operatorname{Prob}(\mathrm{SAE})$ & $(95 \% \mathrm{CI})$ & Prob(SAE) & $95 \% \mathrm{CI}$ \\
\hline 5000 & 0.0003 & $0 \cdot 0001-0.0007$ & 0.0003 & $0 \cdot 0001-0 \cdot 0007$ & 0.0005 & $0 \cdot 0002-0 \cdot 0012$ & 0.0002 & $0 \cdot 0001-0 \cdot 0004$ \\
\hline 10000 & 0.0018 & $0.0001-0.0037$ & 0.0016 & $0.0001-0.0033$ & 0.0028 & $0 \cdot 0002-0.0057$ & 0.0009 & $0.0002-0.0020$ \\
\hline 20000 & 0.010 & $0.003-0 \cdot 017$ & 0.0087 & $0.0025-0.0149$ & 0.0153 & $0 \cdot 0045-0.0261$ & 0.0050 & $0.0003-0.0098$ \\
\hline 30000 & 0.03 & $0.01-0.04$ & 0.023 & $0.011-0.036$ & 0.041 & $0 \cdot 019-0.062$ & 0.014 & $0.003-0.025$ \\
\hline 40000 & 0.05 & $0.03-0.08$ & 0.05 & $0.03-0.07$ & 0.08 & $0.05-0.34$ & 0.03 & $0.69-0.05$ \\
\hline 50000 & 0.09 & $0 \cdot 06-0 \cdot 12$ & 0.08 & $0 \cdot 05-0 \cdot 11$ & $0 \cdot 13$ & $0 \cdot 08-0 \cdot 18$ & 0.05 & $0.01-0.08$ \\
\hline 100000 & $0 \cdot 37$ & $0 \cdot 25-0.49$ & $0 \cdot 32$ & $0 \cdot 20-0 \cdot 44$ & $0 \cdot 45$ & $0.31-0.59$ & $0 \cdot 21$ & $0.08-0.35$ \\
\hline 150000 & 0.62 & $0 \cdot 46-0.78$ & 0.56 & $0 \cdot 38-0.74$ & 0.69 & $0 \cdot 54-0.85$ & $0 \cdot 42$ & $0 \cdot 19-0.66$ \\
\hline 200000 & 0.77 & $0.62-0.92$ & $0 \cdot 72$ & $0.55-0.90$ & 0.82 & $0.69-0.95$ & 0.61 & $0.35-0.85$ \\
\hline
\end{tabular}

Prob(SAE): Individual probability of developing a severe adverse event (\%); CI: confidence interval. 
Male

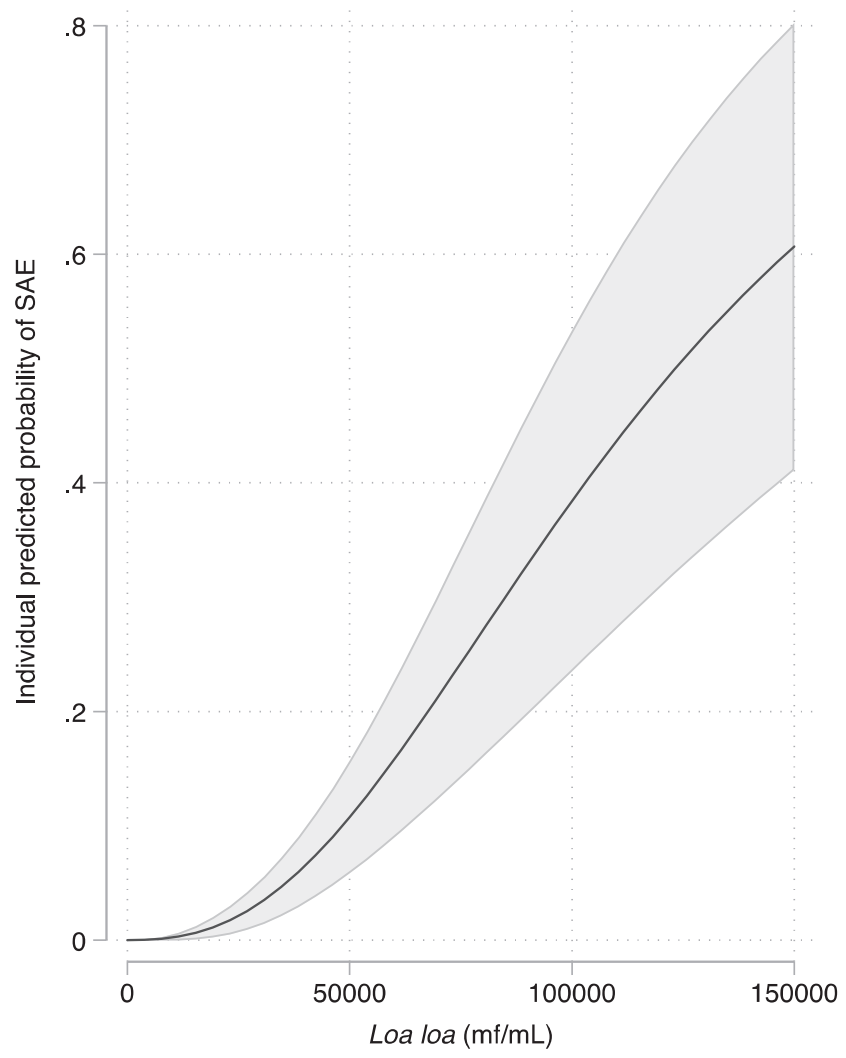

Female

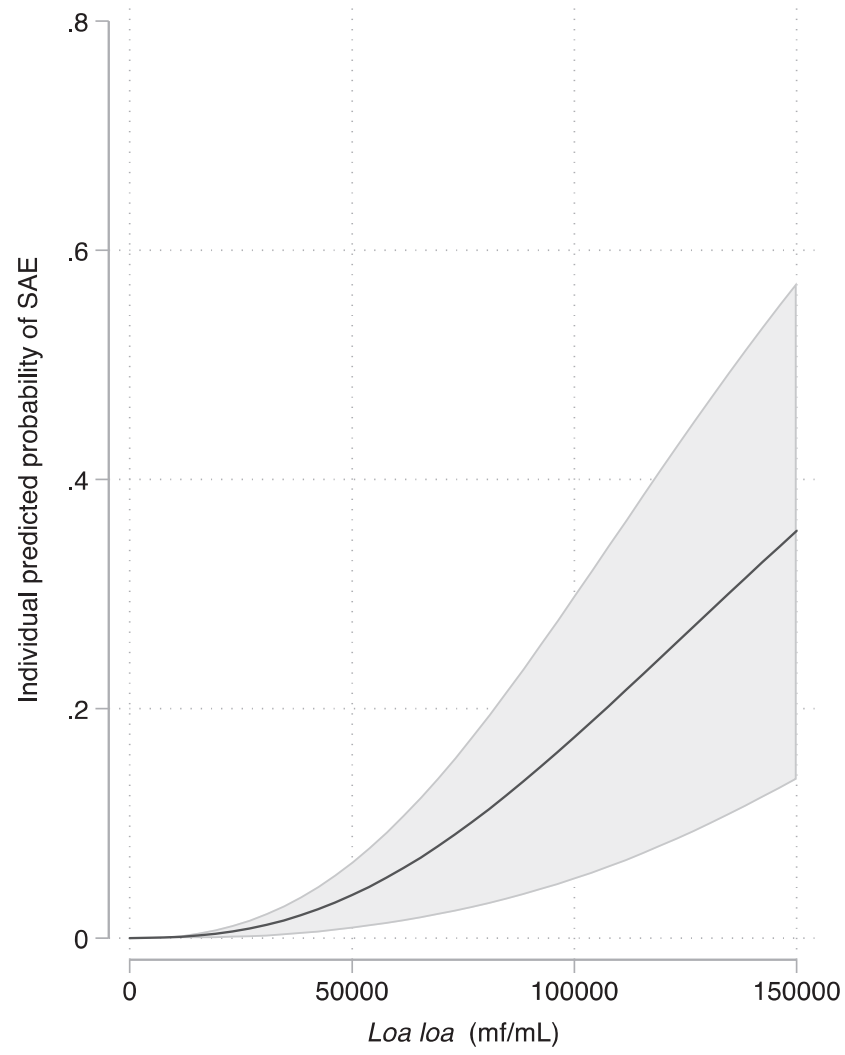

Fig. 2. Predicted probabilities of developing a post-ivermectin of severe adverse event according to the individual $L$ loa microfilarial density, according to the sex.

Table 4

Misclassification estimation after bootstrap procedures applied in the multivariable fractional polynomial models to estimate the probability of developing a serious adverse event.

\begin{tabular}{|c|c|c|c|c|}
\hline & Model with $L$ loa MFD alone & Adjusted model with sex & Adjusted model with age and sex & Full adjusted mode \\
\hline \multicolumn{5}{|c|}{ Absolute count (No of cases) } \\
\hline Median & $-0 \cdot 13$ & $-0 \cdot 18$ & 0.05 & $0 \cdot 22$ \\
\hline Interquartile range & -3.01 to 2.99 & $-2 \cdot 74$ to $3 \cdot 00$ & -2.90 to 3.00 & $-2 \cdot 56$ to $3 \cdot 21$ \\
\hline $0.025-0.975$ deciles & -8.05 to 7.87 & $-8 \cdot 36$ to 8.41 & -8.57 to 8.30 & -7.63 to 8.32 \\
\hline \multicolumn{5}{|c|}{ Percentage variation (\%) } \\
\hline Median & -0.91 & $-1 \cdot 33$ & $0 \cdot 45$ & $1 \cdot 75$ \\
\hline Interquartile range & $-25 \cdot 32$ to $19 \cdot 40$ & -23.58 to 19.02 & $-23 \cdot 71$ to $19 \cdot 05$ & $-21 \cdot 28$ to $21 \cdot 22$ \\
\hline 0.025-0.975 deciles & $-85 \cdot 38$ to $43 \cdot 13$ & -96.65 to 44.73 & $-101 \cdot 00$ to $45 \cdot 11$ & -80.59 to 46.97 \\
\hline
\end{tabular}

context of these pilot clinical trials during which evidence of postIVM SAEs has emerged, the hospitalization of this patient could have been precautionary, and may not necessarily reflect a definitive case of SAE.

Our models predict that nearly $10 \%$ of people with 50,000 L loa $\mathrm{mf} / \mathrm{mL}$ and about one third of those with $100,000 \mathrm{mf} / \mathrm{mL}$ will develop an SAE after IVM treatment. Identification of cofactors explaining why some individuals with a given MFD will develop an SAE while others will not, could help prevent SAE cases. No association was found between the occurrence of SAEs and infection with a simian strain of $L$ loa [9]. Coinfection with Plasmodium falciparum does not seem to play a role [6], but this result requires further investigation

Table 5

$\beta$-coefficients after 0.632 bootstrap procedures applied in the multivariable fractional polynomial models to estimate the probability of developing a serious adverse event.

\begin{tabular}{|c|c|c|c|c|}
\hline & $\begin{array}{l}\text { Model with } L \text { loa MFD alone } \\
\text { Coefficients (median and IQR) }\end{array}$ & $\begin{array}{l}\text { Adjusted model with sex } \\
\text { Coefficients (median and IQR) }\end{array}$ & $\begin{array}{l}\text { Adjusted model with age and sex } \\
\text { Coefficients (median and IQR) }\end{array}$ & $\begin{array}{l}\text { Full adjusted model } \\
\text { Coefficients (median and IQR) }\end{array}$ \\
\hline $\operatorname{Loa}^{F P}$ & $2 \cdot 57(2 \cdot 41-2 \cdot 75)$ & $2 \cdot 51(2 \cdot 36-2 \cdot 70)$ & $2 \cdot 54(2 \cdot 37-2 \cdot 72)$ & $2 \cdot 62(2 \cdot 45-2 \cdot 80)$ \\
\hline $\operatorname{Age}_{1}^{F P}$ & & & $-2 \cdot 62(-4 \cdot 01$ to $-1 \cdot 36)$ & $-2 \cdot 37(-3.88$ to $-1 \cdot 04)$ \\
\hline $\operatorname{Age}_{2}{ }^{F P}$ & & & $15 \cdot 95(10 \cdot 76-22 \cdot 28)$ & $17 \cdot 76(12 \cdot 45-23 \cdot 63)$ \\
\hline Sex & & $1 \cdot 16(0 \cdot 95-1 \cdot 39)$ & $1 \cdot 08(0 \cdot 84-1 \cdot 32)$ & $1 \cdot 22(1 \cdot 00-1 \cdot 47)$ \\
\hline Mansonella $_{1}^{F P}$ & & & & $0.02(-0.004-0.05)$ \\
\hline Mansonella $_{2}^{F P}$ & & & & $-0.27(-10 \cdot 68$ to 0.32$)$ \\
\hline
\end{tabular}

IQR: interquartile range; FP: fractional polynomials; 0.632 bootstrap procedures using 1000 iterations. 
because the retinal lesions observed in patients with cerebral malaria and Loa-related encephalopathy are very similar [8]. Other cofactors such as coinfection with other pathogens such as nematodes (especially Strongyloides stercoralis) and trematodes, which were not assessed during the trials, could favour the development of Loarelated post-IVM SAEs and careful examination of the individual "Serious adverse experience reports" sent by the national programmes to the Mectizan Donation Program might help identify some of them. It might also be instructive to explore factors influencing the pharmacokinetics of IVM. Drug-drug interactions could play a role [10], but no information regarding concomitant treatment was collected during the two trials used to develop our predictive models. The clinical condition associated with Loa-related encephalopathy differs markedly from that observed in animals or humans treated with toxic doses of IVM, or in animals (especially dogs) carrying a mutation in the multidrug resistance $(m d r-1)$ gene that makes the blood-brain barrier defective and allows IVM to accumulate in brain tissue [11]. In the latter cases, the main signs and symptoms are salivation, vomiting, ataxia, tremors, mydriasis, and confusion [12,13]. A pilot study comparing the $m d r-1$ genotypes in four subjects who had experienced a post-IVM $L$ loa-related SAE and nine matched non-SAE controls showed no loss-of-function mutation in any subject, but haplotypes associated with altered drug disposition were present as homozygotes in two of the SAE patients [14]. Additional pharmacogenetics studies could help identify a genetic predisposition to develop a post-IVM SAE.

In this study, we used $L$ loa MFD measured by microscopic examination of calibrated ( $50 \mu \mathrm{L})$ TBS to estimate the individual risk of developing an SAE. A field-friendly mobile phone video microscope (LoaScope), measuring $L$ loa MFD within two minutes, was developed and its performance evaluated as part of the TNT study [2]. The MFD results obtained by TBS examination and LoaScope are highly correlated but as the LoaScope examines only $13 \cdot 7 \mu \mathrm{L}$ of blood, this correlation decreases as the $L$ loa MFD decreases, and infection can be missed in case of very low MFD $(<500 \mathrm{mf} / \mathrm{mL})$. The LoaScope has been used in various countries to assess the prevalence of $L$ loa as well as the MFD distribution in the population [15,16], and could become the standard method used by onchocerciasis (and possibly lymphatic filariasis) elimination programmes. The formulas we developed in this study for TBS-based $L$ loa MFD can easily be adapted to the performance characteristics of the LoaScope.

Concerning the robustness of our model, the semi-external validation allows us to be reassured with very low median deviations for each of the models. Moreover, the coefficients obtained with the 0.632 Bootstrap procedure are very close to our basic models.

In conclusion, this study provides programme managers and decision-makers with a practical tool to accurately predict the risk of post-IVM SAEs in a setting where onchocerciasis and loiasis are coendemic, allowing them to determine the most suitable and costeffective treatment strategies for the target population. Finally, this tool can also be useful for practitioners treating individual cases of loiasis, in guiding medical decisions surrounding choice of treatment.

\section{Data sharing statement}

The de-identified dataset may be obtained from the Institut de Recherche pour le Développement by emailing to the corresponding author. Prior to releasing any data there must be a signed data access agreement and an approved analysis plan.

\section{Contributors}

JG, NGW, JFD, JK and MB conducted the clinical trials. CBC developed, performed, and interpreted the statistical analyses. $C B$ developed and performed the bootstrap analyses. $\mathrm{CBC}$ wrote the first version of the manuscript. All authors critically reviewed and approved the final version of the manuscript.

\section{Declaration of Competing Interest}

We declare no competing interests.

\section{Acknowledgments}

We are grateful to the Cameroonian authorities for their help and support. We are also grateful to the populations for their participation. We thank Charlie Whittaker for his technical advices and reading, and Annette Kuesel for her expertise and reading.

\section{Funding}

This work was supported by the UNDP/World Bank/WHO Special Programme for Research and Training in Tropical Diseases; the Institut de Recherche pour le Développement; the Mectizan Donation Program; and the Bill \& Melinda Gates Foundation.

\section{Supplementary materials}

Supplementary material associated with this article can be found, in the online version, at doi:10.1016/j.eclinm.2020.100582.

\section{Reference}

[1] Gardon J, Gardon-Wendel N, Demanga-Ngangue Kamgno J, Chippaux JP, Boussinesq $M$. Serious reactions after mass treatment of onchocerciasis with ivermectin in an area endemic for Loa loa infection. Lancet 1997;350:18-22.

[2] Kamgno J, Pion SD, Chesnais CB, et al. A test-and-not-treat strategy for onchocerciasis in Loa loa-endemic areas. N Engl J Med 2017;377:2044-52.

[3] Boussinesq M, Fobi G, Kuesel AC. Alternative treatment strategies to accelerate the elimination of onchocerciasis. Int Health 2018;10:i40-8.

[4] Lenk EJ, Moungui HC, Boussinesq M, et al. A test-and-not-treat strategy for onchocerciasis elimination in Loa loa co-endemic areas: cost analysis of a pilot in the Soa health district, Cameroon. Clin Infect Dis 2019;70:1628-35.

[5] World Health Organization. Report of the second Meeting of the WHO Onchocerciasis Technical Advisory Subgroup, WHO, Geneva, Switzerland, 12-14 February 2018. WHO/CDS/NTD/PCT/2018.11. 2018 https://apps.who.int/iris/bitstream/ handle/10665/277238/WHO-CDS-NTD-PCT-2018.11-eng.pdf.

[6] Fokom-Domgue J, Pion SD, Gounoue R, et al. Absence of an association between Plasmodium falciparum infection and post-ivermectin Loa-related non-neurologic serious adverse events. Am J Tropical Medicine Hyg 2014;90:335-8.

[7] Anonymous. Central nervous system (CNS) complications of loiasis and adverse events following treatment. Report of an invited consultation: 2-3 October 1995. 1996.

[8] Fobi G, Gardon J, Santiago M, Ngangue D, Gardon-Wendel N, Boussinesq M. Ocular findings after ivermectin treatment of patients with high Loa loa microfilaremia. Ophthalmic Epidemiol 2000;7:27-39.

[9] Kamgno J, Pion SD, Mackenzie CD, Thylefors B, Boussinesq M. Loa loa microfilarial periodicity in ivermectin-treated patients: comparison between those developing and those free of serious adverse events. Am J Trop Med Hyg 2009:81:1056-61.

[10] Chandler RE. Serious neurological adverse events after ivermectin - Do they occur beyond the indication of onchocerciasis? Am J Trop Med Hyg 2018;98:382-8.

[11] Mealey KL, Bentjen SA, Gay JM, Cantor GH. Ivermectin sensitivity in collies is associated with a deletion mutation of the mdr1 gene. Pharmacogenetics 2001;11:727-33.

[12] Paul AJ, Tranquilli WJ, Seward RL, Jr Todd KS, DiPietro JA. Clinical observations in collies given ivermectin orally. Am J Vet Res 1987:48:684-5.

[13] Woodward KN. Toxicity in animals: target species. Curr Pharm Biotechnol 2012;13:952-68.

[14] Bourguinat C, Kamgno J, Boussinesq M, Mackenzie CD, Prichard RK, Geary TG. Analysis of the mdr-1 gene in patients co-infected with Onchocerca volvulus and Loa loa who experienced a post-ivermectin serious adverse event. Am J Trop Med Hyg 2010;83:28-32.

[15] Emukah E, Rakers LJ Kahansim B, et al. In Southern Nigeria Loa loa blood microfilaria density is very low even in areas with high prevalence of loiasis: results of a survey using the new LoaScope technology. Am J Trop Med Hyg 2018;99:116-23.

[16] Pion SD, Nana-Djeunga H, Niamsi-Emalio Y, et al. Implications for annual retesting after a test-and-not-treat strategy for onchocerciasis elimination in areas coendemic with Loa loa infection: an observational cohort study. Lancet Infect Dis 2020;20:102-9 